Article

\title{
Sea Buckthorn Hippophae rhamnoides and Fruit Flies Rhagoletis batava: Search for Volatile Semiochemicals Involved in Pest Attraction
}

\author{
Laima Blažytė-Čereškienė *, Vincas Būda, Violeta Apšegaitè, Sandra Radžiutè, Jurga Būdienė, \\ Dominykas Aleknavičius and Raimondas Mozūraitis $\mathbb{D}$
}

check for updates

Citation: Blažytė-Čereškienè, L.; Būda, V.; Apšegaitè, V.; Radžiutè, S.; Būdienè, J.; Aleknavičius, D.;

Mozūraitis, R. Sea Buckthorn

Hippophae rhamnoides and Fruit Flies Rhagoletis batava: Search for Volatile Semiochemicals Involved in Pest Attraction. Horticulturae 2022, 8, 179. https://doi.org/10.3390/

horticulturae 8020179

Academic Editors: Katarzyna Kmieć, Elżbieta Mielniczuk and Katarzyna Golan

Received: 2 February 2022

Accepted: 18 February 2022

Published: 21 February 2022

Publisher's Note: MDPI stays neutral with regard to jurisdictional claims in published maps and institutional affiliations.

Copyright: () 2022 by the authors Licensee MDPI, Basel, Switzerland. This article is an open access article distributed under the terms and conditions of the Creative Commons Attribution (CC BY) license (https:// creativecommons.org/licenses/by/ $4.0 /)$.
Laboratory of Chemical and Behavioural Ecology, Institute of Ecology, Nature Research Centre, Akademijos str. 2, LT-08412 Vilnius, Lithuania; vincas.buda@gamtc.lt (V.B.); violeta.apsegaite@gamtc.lt (V.A.); sandra.radziute@gamtc.lt (S.R.); jurga.budiene@gamtc.lt (J.B.); dominykas.aleknavicius@gamtc.lt (D.A.); raimondas.mozuraitis@gamtc.lt (R.M.)

* Correspondence: laima.blazyte@gamtc.lt; Tel.: +370-68584622

\begin{abstract}
Rhagoletis batava (Diptera: Tephritidae), is the most important pest of Hippophae rhamnoides fruits. For detection and monitoring of R. batava, traps supplied with nonspecific attractants are used. Thus, new, more specific attractants for environment-friendly pest control are needed. Such attractants could be fruit-related semiochemicals that are involved in the host location by flies. Behavioural Y-olfactometer tests revealed that $R$. batava males were attracted to ripe fruit odour, while females preferred unripe and semi-ripe fruits. Thermal desorption gas chromatography-mass spectrometry (GC-MS) analysis revealed substantial quantitative and qualitative changes in volatiles between unripe and ripe fruits. In the unripe fruit emission, 41 volatile compounds were isolated, whereas 64 compounds were sampled from the ripe fruits. The total amount of volatiles increased five times during the fruit ripening. Gas chromatography-electroantennogram detection (GC-EAD) and GC-MS analyses of the fruit headspace volatiles revealed at least 26 compounds in unripe and 27 compounds in ripe fruits eliciting antennal responses of $R$. batava for both sexes. The fruits of these two ripening stages differed qualitatively in the single EAD-active compound only, i.e., 3-methylbutyl 2-methylpropionate. Esters were the most abundant volatiles, composing $84 \%$ and $93 \%$ of EAD-active compounds in the emissions of unripe and ripe fruits, respectively. Based on the persistent EAG responses, 17 compounds were selected as the most promising candidates for kairomone attractants of the sea buckthorn pest $R$. batava.
\end{abstract}

Keywords: sea buckthorn; fruits; volatiles; Rhagoletis batava; Diptera; Tephritidae; electroantennography; semiochemicals; pest management; behaviour modification

\section{Introduction}

Sea buckthorn, Hippophae rhamnoides L. (Rosales: Elaeagnaceae) is a fruit-bearing bush naturally distributed in Northern and Central Europe, Caucasus, and Asia [1]. Sea buckthorn is cultivated in many European countries, Canada, Russia, and China as a highly valuable plant [2,3]. Since a few decades ago, this plant has attracted considerable attention from researchers around the world, mainly for its nutritional and medical properties: richness in minerals, vitamins, polysaccharides, unsaturated fatty acid, terpenoids, polyphenolic and nonsteroidal compounds, flavonoids, and volatile components [4,5]. Sea buckthorn fruits as a raw material are used for various purposes in the food, medicine, and cosmetic industries [2]. As the demand for sea buckthorn raw material in the global market rises, the area of sea buckthorn plantations increases every year [6].

During recent years, the sea buckthorn fruits have become more and more damaged by the fruit fly Rhagoletis batava Hering (Diptera: Tephritidae), an insect pest that began to spread rapidly in central and eastern European countries [7-9]. In the years of mass 
development of $R$. batava flies and without applying any pest control means, fruit harvest losses can reach near $100 \%$ [10]. This problem needs to be solved very urgently, as the infestation of sea buckthorn flies in sea buckthorn plantations has reached a level where not only the establishment of new plantations is declining, but the destruction of existing ones starts to take place as well [6].

Insecticides are used to control pest insects; however, those are not among the most suitable means, due to the life peculiarities of the insect, most of the pre-imaginal stages passes within fruits; moreover, insecticide application can lead to environmentally detrimental consequences. For detection and monitoring of $R$. batava fruit flies, yellow sticky traps supplied with ammonium carbonate or ammonium acetate lures are recommended [11]. However, ammonia-based attractants are nonspecific; they also attract non-target insects that can clutter traps [12,13]. Recently, an aggregation pheromone of $R$. batava was revealed [14]; however, its applicability in pest control is not yet known. Thus, new, more specific attractants for environment-friendly pest control are still needed, especially considering organic (ecological) farming.

Most of the phytophagous insects search for their host-plants by olfactory cues, i.e., volatile molecules emitted from potential host-plants [15]. These volatile compounds play an important role in regulating the behaviour of herbivorous insects, such as orientation, nutrition, mate location, and oviposition [16-18]. Volatile molecules released from sea buckthorn fruits might be of vital importance for fruit fly $R$. batava to detect the host. Unfortunately, to our knowledge, no fruit-related semiochemicals have been identified for $R$. batava species. As fruit damaged by R. batava can already be observed in the early stage of buckthorn fruit ripening (Aleknavičius, pers. obs.), the volatiles emitted during this stage could be important for the fruit fly to detect a suitable host-plant. The goal of this study was to compare volatiles released by ripe and unripe $H$. rhamnoides fruits, as well as to determine at which ripening stage the fruits were preferred by $R$. batava fruit flies, and to identify semiochemicals that could mediate host-plant location by the flies. Data on such volatile compounds could lead to the application of their synthetic analogues for both pest population monitoring and as a potential tool for pest insect control.

\section{Materials and Methods}

\subsection{Insects}

For laboratory assays, sea buckthorn fruit flies, R. batava, were collected in May as puparia in soil under sea buckthorn shrubs in an organic plantation (GPS coordinates: $55^{\circ} 15^{\prime} 12.179^{\prime \prime} \mathrm{N}, 25^{\circ} 26^{\prime} 23.049^{\prime \prime}$ E) in Stacijava village, Molètai district, Lithuania. Each puparium was placed individually in a $14 \mathrm{~mL}$ glass vial with wet $3 \mathrm{~cm}^{2}$ filter paper inside and plugged by a foam stopper. The vials were kept in a climate chamber Fitotron (Weiss Gallenkamp, Loughborough, UK) under 20-24 ${ }^{\circ} \mathrm{C}, 16 \mathrm{~L}: 8 \mathrm{D}$ (light:dark) photoperiod, and $65-75 \%$ relative humidity. A few drops of water were added to a filter paper weekly to keep humidity inside a vial. After the emergency, the flies were sexed based on the presence or absence of an ovipositor. In the same individual vials, males and females were kept separately under $18-20{ }^{\circ} \mathrm{C}$, natural daylight photoperiod, 50-60\% relative humidity, and fed on $10 \%$ sugar solution in water.

\subsection{Sea Buckthorn Fruits}

H. rhamnoides fruits were sampled from the same sea buckthorn plantation as insect cocoons. The fruits were collected at three ripening stages: at the beginning of fruit ripening, i.e., unripe fruit (green soft) in July, semi-ripe (yellow) at the end of July, and fully ripe fruit (orange) at the end of August in 2020-2021. Collected fruit samples were immediately transferred to the laboratory for behavioural tests and volatiles sampling.

\subsection{Evaluation of the Host-Plant Odour Attractiveness Using Y-Tube Olfactometer}

To test fruit fly $R$. batava preference to volatiles emitted by sea buckthorn fruits at three ripening stages, a Y-tube olfactometer (length of the main tube- $25 \mathrm{~cm}$; length of arms- 
$17 \mathrm{~cm}$; embranchment angle- $110^{\circ}$; inner diameter $-5 \mathrm{~cm}$ ) with an air supply system CADS-4CPP (Sigma Scientific LLC, Micanopy, FL, USA) was used. Activated charcoal filtered and humidified air at a rate of $0.7 \mathrm{~L} / \mathrm{min}$ was pushed through each $300 \mathrm{~mL}$ glass flask containing odour sources. The odour source was 30 sea buckthorn fruits on a twig (without leaves). Fruits of the three ripening stages (unripe, semi-ripe, and ripe fruits) were tested separately. A single fly was released into the $Y$ olfactometer at the end of the main tube. The choice duration for a fly was $10 \mathrm{~min}$ and was recorded when the fly reached the upwind end of the arm-tube. Adults that did not make a choice were not included in the analysis. Only 4-15 days old adults (males and females) were used (lifespan about 30 days). Males and females were fed on the sucrose solution before the testing. The tests were carried out at $23 \pm 2{ }^{\circ} \mathrm{C}$, from 09.00 until $18.00 \mathrm{~h}$.

Before changing the odour source, the olfactometer was disassembled, washed with hexane, soaked in distilled water, and heated to $160^{\circ} \mathrm{C}$ in a thermostat. The silicone parts of the olfactometer were washed with hexane, soaked in distilled water overnight, and dried at room temperature.

The absence of any positional preference of the arms was verified by testing the flies without any odour. No significant preference was recorded in this preliminary test. Moreover, to avoid any positional bias, the position of the Y-tube arms connected to the odour chambers was changed, i.e., the arms were swapped, after testing 5 adults.

\subsection{Sampling and Analysis of Fruit-Released Volatiles}

To compare the amounts of the compounds emitted by ripe and unripe H. rhamnoides fruits, forty grams of freshly picked sea buckthorn fruits were placed in a $300 \mathrm{~mL}$ Erlenmeyer flask and closed with a ground-glass stopper with an air inlet and outlet connected to the airflow system. Five microliters of 2-phenyethyl acetate as internal standard (concertation $1 \mathrm{mg} / \mathrm{mL}$ in hexane) were introduced to all blank (control) and sample flasks. C2-BXXX-5201 Biomonitoring glass tubes (Markers International Ltd., Bridgend, UK) with a compound absorption range from $C_{3}$ to $C_{30}$ were used. Pre-conditioned desorption glass tubes were attached to the air outlet part with silicone hoses and volatiles released throughout the experiments were trapped in them. Collection of volatiles was carried out for $2 \mathrm{~h}$ with an airflow (charcoal filtered) rate of $500 \mathrm{~mL} / \mathrm{min}$. Afterwards, the adsorption glass tubes were sealed with caps and kept at $4{ }^{\circ} \mathrm{C}$ in a refrigerator until desorption and GC-MS analyses. A sampling of volatiles from unripe and ripe fruits was carried out in triplicate. An empty Erlenmeyer flask was used twice as a control to detect volatiles in the laboratory environment.

Desorption of analytes was performed in an automated TD-20 Thermo desorption unit (Shimadzu, Japan) coupled to GC-MS (GCMS-QP2020 ultra, Shimadzu, Kyoto, Japan) at $250{ }^{\circ} \mathrm{C}(10 \mathrm{~min})$ at helium flow of $70 \mathrm{~mL} / \mathrm{min}$. Volatiles released from the sample tubes were reabsorbed on a trap tube kept isothermal at $-10^{\circ} \mathrm{C}$ and then transferred on GC polar column (DB-WAX, $30 \mathrm{~m} \times 0,25 \mathrm{~mm} \times 0,25 \mu \mathrm{m}$ ). GC-MS analyses of collected sea buckthorn fruit volatiles were performed on the same conditions as described above for GC-EAD and GC-MS, except that the oven temperature was programmed isothermally at $40^{\circ} \mathrm{C}$ for $1 \mathrm{~min}$; afterward, it was raised to $240^{\circ} \mathrm{C}$ at a rate of $5{ }^{\circ} \mathrm{C} / \mathrm{min}$ and then maintained isothermally for $1 \mathrm{~min}$. The injector was set at $34^{\circ} \mathrm{C}$.

\subsection{Sampling Volatiles for Olfactory Activity Evaluation}

To reveal the $H$. rhamnoides fruit emitted compounds that were olfactorily active to fruit fly R. batava, the headspace volatiles were collected from $180 \mathrm{~g}$ fruits (either unripe or ripe) placed into a $500 \mathrm{~mL}$ glass flask fitted with an inlet for purified air supplied at $0.5 \mathrm{~L} / \mathrm{min}$ flow and with an outlet housing adsorbent trap filled with $150 \mathrm{mg}$ of Tenax TA 60-80 mesh, (Supelco, Darmstadt, Germany) in a Pasteur glass pipette. For the collection of control samples, a charcoal-filtered airstream was pulled through an empty glass flask. Volatiles were collected for $24 \mathrm{~h}$, afterward eluted with $300 \mu \mathrm{L}$ of diethyl ether, and concentrated to $300 \mu \mathrm{L}$ under a gentle nitrogen stream. Samples from three collections were combined and 
stored at $-18{ }^{\circ} \mathrm{C}$ until gas chromatography-electroantennogram detection (GC-EAD) and gas chromatography-mass spectrometry (GC-MS) analyses.

\subsection{GC-EAD Recording}

Coupled GC-EAD was performed using a Clarus 500 gas chromatograph (Perkin Elmer, Waltham, MA, USA) equipped with a DB-Wax capillary column $(30 \mathrm{~m} \times 0.25 \mathrm{~mm}$ $\times 0.25 \mu \mathrm{m}$; Agilent Technologies, Santa Clara, CA, USA). The oven temperature was maintained isothermally at $40^{\circ} \mathrm{C}$ for $1 \mathrm{~min}$; afterward, the temperature was raised to $200{ }^{\circ} \mathrm{C}$ at a rate of $5{ }^{\circ} \mathrm{C} / \mathrm{min}$, then increased to $240{ }^{\circ} \mathrm{C}$ at a rate of $10{ }^{\circ} \mathrm{C} / \mathrm{min}$, and afterwards maintained isothermally for $11 \mathrm{~min}$. Hydrogen at the flow rate of $1.5 \mathrm{~mL} / \mathrm{min}$ was used as a carrier gas. The injector and the detector temperatures were set isothermal at $240{ }^{\circ} \mathrm{C}$. At the end of the column, the GC effluent was split into two equal parts: one stream was directed to the flame ionization detector (FID) and the other stream flowed to the EAD detector. A nitrogen make-up gas at the flow rate of $5 \mathrm{~mL} / \mathrm{min}$ was used to enhance FID performance. The EAD effluent was delivered into a stream of purified and humidified air $(0.5 \mathrm{~m} / \mathrm{s})$ over the antennal preparation.

The flies used in GC-EAD analyses were not chilled or anaesthetized before use. Glass capillary electrodes filled with Ringer solution (Fresenius Kabi, Warsaw, Poland) and grounded via a silver wire were used. The indifferent electrode was inserted in the severed head of the fly, while the recording electrode, connected to a high-impedance DC amplifier with automatic baseline drift compensation, was brought into contact with the distal end of the fly antenna. The antennal signal was amplified 10 times, converted to a digital signal by IDAC-4, and recorded simultaneously with the FID signal on a computer using GcEad V. 4.4 (Synthech, NL 1998). Four successful recordings of male antennae and five of female antennae were used for the GC-EAD analysis.

\subsection{Identification of Olfactory Active Fruit-Released Volatiles}

The headspace volatile samples were analysed on a Shimadzu GC-2010 coupled with Shimadzu MS-QP 2010 Plus mass selective detector (Shimadzu, Kyoto, Japan). The GC was equipped with a DB-Wax capillary column $(30 \mathrm{~m} \times 0.25 \mathrm{~mm} \times 0.25 \mu \mathrm{m}$; Agilent Technologies, Santa Clara, CA, USA) and operated under the same conditions as described above for GC-EAD, except that helium was used as the carrier gas at the flow rate of $1.5 \mathrm{~mL} / \mathrm{min}$. Electron ionization spectra were acquired at an electron energy of $70 \mathrm{eV}$; the interface and ion source temperatures were held isothermal at $250{ }^{\circ} \mathrm{C}$. The EAD active compounds were tentatively identified by comparison of their mass spectral data and retention indexes with the NIST version 2.0 mass spectra search program (National Institute of Standards and Technology, Gaithersburg, Maryland, USA); the retention times and mass spectra of identified compounds were confirmed through injecting synthetics under the same GC-MS program as described above.

\subsection{Chemicals}

The following compounds were purchased from Sigma-Aldrich (St. Louis, MO, USA): ethyl 2-methylbutanoate (99\% chemical purity), ethyl 3-methyl-2-butenoate (98\% chemical purity), 3-methylbutyl 2-methylbutanoate ( $\geq 98 \%$ chemical purity), 3-methylbutyl 3methylbutanoate ( $\geq 98 \%$ chemical purity), propyl hexanoate ( $\geq 90 \%$ chemical purity), ethyl octanoate ( $\geq 99 \%$ chemical purity), ethyl benzoate ( $\geq 99 \%$ chemical purity), 3-methylbutyl benzoate ( $\geq 98 \%$ chemical purity), ( $E$ )- $\beta$-ocimene ( $\geq 90 \%$ chemical purity), 3-methylbutan1 -ol ( $\geq 99 \%$ chemical purity), 1-octen-3-ol (98\% chemical purity), (E)-geranyl acetone ( $96 \%$ chemical purity), hexane ( $\geq 99 \%$ chemical purity). Ethyl butanoate ( $99 \%$ chemical purity), ethyl 3-methylbutanoate ( $98 \%$ chemical purity), 3-methylbutyl 2-methylpropionate $(99 \%$ chemical purity), ethyl hexanoate ( $99 \%$ chemical purity), ethyl heptanoate $(99 \%$ chemical purity) were purchased from Alfa Aesar (Ward Hill, MA, USA). 


\subsection{Statistical Analysis}

The statistical analysis was performed using the software Statistica 6.0 (StatSoft, Tulsa, OK, USA). The behavioural results in the Y-tube olfactometer were analysed using the chisquare test. To compare amounts of volatile compounds in ripe and unripe fruit emissions, the amounts of volatiles collected in control were withdrawn from amounts collected from fruit samples. A nonparametric Mann-Whitney U test was applied to evaluate differences in volatile amounts of ripe and unripe fruit volatiles.

\section{Results}

\subsection{Behavioural Responses of Rhagoletis batava to Sea Buckthorn Fruits}

Before recording of volatiles' effect, the blank test in a Y-tube olfactometer was performed. There was no difference in the choice made by the fruit flies to each arm $(p>0.05)$, indicating that the olfactometry was not biased (Figure 1).
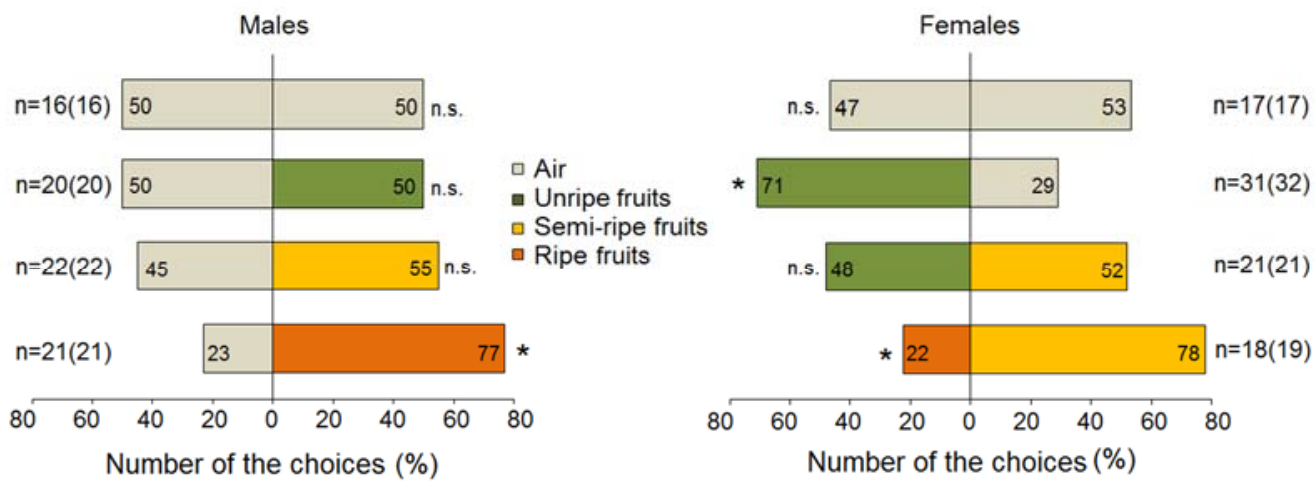

Figure 1. Choices by Rhagoletis batava between the odours of sea buckthorn fruits depending on their ripening stage in Y-tube olfactometer. $n$, Number of flies that made choice between two arms of Y-tube; a total number of flies tested is given in parentheses; ${ }^{*}$ asterisks indicate statistically significant differences between choices $(p<0.05)$; n.s., nonsignificant, $\chi^{2}$ test.

Males of R. batava showed no preference for the volatiles of unripe or semi-ripe fruits over control (pure air) $(p>0.05)$; however, the males preferred the odour of ripe fruits to clean air $(p=0.016)$ (Figure 1$)$. In contrast to males, the females preferred the odour of unripe fruits to pure air $(p<0.05)$ (Figure 1$)$.

The other two tests were designed to determine which ripening stage of the sea buckthorn fruits was preferred by R. batava females. The females demonstrated no preference for unripe fruits over semi-ripe fruits $(p>0.05)$ (Figure 1$)$, whereas the females preferred the odour of semi-ripe fruits to the odour of ripe fruits $(p=0.018)$.

\subsection{Volatiles of Sea Buckthorn Fruits at Two Ripening Stages}

In the emissions of unripe fruits, 41 volatile compounds were isolated, whereas 64 compounds were sampled from the headspace of ripe fruits. The total amount of volatiles increased five times, i.e., from 485 to $2588 \mathrm{ng} / \mathrm{g} / \mathrm{h}$ in the emissions of unripe and ripe fruits, respectively (Table 1). The volatile blend of unripe fruits comprised 21 esters, 5 alcohols, 4 ketones, 3 of each aldehyde, terpenes, and volatile fatty acids, and 1 of each alkane and unidentified compound. Thirty-six esters, 12 alcohols, 7 terpenes, 3 aldehydes, 3 unknown compounds, 2 ketones, 2 volatile fatty acids, and 1 alkane composed the volatile blend of ripe fruits (Figure 2). Thirteen compounds were unique for unripe fruits. Three of these compounds i.e., (Z)-3-hexanal, $(E)$-2-hexanal, and (Z)-3-hexenyl acetate were detected at concentrations of higher than $5 \mathrm{ng} / \mathrm{g} / \mathrm{h}$ (Table 1 ). 
Table 1. Volatile compounds identified in the headspace of sea buckthorn Hippophae rhamnoides fruits at two ripening stages: unripe fruits and ripe fruits.

\begin{tabular}{|c|c|c|c|c|c|}
\hline No & Compound & $\begin{array}{l}\text { Compound } \\
\text { Group }\end{array}$ & RT & $\begin{array}{l}\text { Unripe Fruits, } \\
\mathrm{ng} / \mathrm{g} / \mathrm{h} \pm \mathrm{SE}^{8}\end{array}$ & $\begin{array}{l}\text { Ripe Fruits, } \\
\text { ng/g/h } \pm \text { SE }\end{array}$ \\
\hline 1 & 3-Methylbutanal & $\mathrm{AL}^{1}$ & 3.46 & $1.54 \pm 0.13$ & \\
\hline 2 & Ethanol & $\mathrm{OH}^{2}$ & 3.50 & & $9.49 \pm 4.80$ \\
\hline 3 & Ethyl 2-methylpropanoate & $\mathrm{ES}^{3}$ & 3.75 & & $49.38 \pm 24.90$ \\
\hline 4 & 2-Pentanone & $\mathrm{KE}^{4}$ & 4.03 & $2.07 \pm 0.31 * 9$ & $12.39 \pm 12.39 *$ \\
\hline 5 & Ethyl butanoate & ES & 4.77 & $13.57 \pm 2.96$ & $114.04 \pm 7.69$ \\
\hline 6 & Ethyl 2-methylbutanoate & ES & 5.05 & $14.45 \pm 5.14$ & $162.23 \pm 63.17$ \\
\hline 7 & Ethyl 3-methylbutanoate & ES & 5.30 & $21.90 \pm 3.97$ & $243.23 \pm 31.19$ \\
\hline 8 & 2-Methylpropan-1-ol & $\mathrm{OH}$ & 5.62 & & $33.50 \pm 8.49$ \\
\hline 9 & 3-Methylbutyl acetate & ES & 6.25 & $4.17 \pm 0.50$ & $21.51 \pm 3.68$ \\
\hline 10 & Ethyl pentanoate & ES & 6.47 & & $3.13 \pm 1.03$ \\
\hline 11 & (Z)-3-Hexanal & AL & 6.61 & $7.63 \pm 0.51$ & \\
\hline 12 & Ethyl 2-butenoate & ES & 7.02 & $1.77 \pm 0.22$ & \\
\hline 13 & $\begin{array}{l}\text { 3-Methylbutyl } \\
\text { 2-methylpropionate }\end{array}$ & ES & 7.93 & & $3.54 \pm 2.06$ \\
\hline 14 & 3-Methylbutan-1-ol & $\mathrm{OH}$ & 8.08 & $57.14 \pm 4.49$ & $230.21 \pm 24.56$ \\
\hline 15 & (E)-2-Hexanal & AL & 8.19 & $5.18 \pm 1.71$ & \\
\hline 16 & Ethyl 3-methyl-2-butenoate & ES & 8.40 & $0.81 \pm 0.18$ & $5.34 \pm 1.46$ \\
\hline 17 & Ethyl hexanoate & ES & 8.66 & $68.17 \pm 17.60$ & $345.52 \pm 30.96$ \\
\hline 18 & $(E)-\beta$-Ocimene & $\mathrm{TE}^{5}$ & 9.06 & $66.45 \pm 7.75$ * & $90.67 \pm 10.51$ * \\
\hline 19 & 3-Methylbutyl butanoate & ES & 9.48 & & $13.76 \pm 0.44$ \\
\hline 20 & 3-Hydroxy-2-butanone (Acetoin) & ES & 9.75 & $37.42 \pm 12.74$ * & $52.97 \pm 9.26 *$ \\
\hline 21 & 3-Methylbutyl 2-methylbutanoate & ES & 9.79 & & $66.53 \pm 19.16$ \\
\hline 22 & 3-Methylbutyl 3-methylbutanoate & ES & 10.13 & $33.27 \pm 2.57$ & $232.16 \pm 16.42$ \\
\hline 23 & $\begin{array}{l}\text { Methyl } \\
\text { 2-hydroxy-2-methylbutanoate }\end{array}$ & ES & 10.30 & $0.02 \pm 0.02$ * & $3.02 \pm 1.60 *$ \\
\hline 24 & (Z)-3-Hexenyl acetate & ES & 10.62 & $9.90 \pm 3.19$ & \\
\hline 25 & Propyl hexanoate & ES & 10.73 & & $9.36 \pm 1.32$ \\
\hline 26 & Ethyl heptanoate & ES & 11.08 & $1.25 \pm 0.13$ & $16.67 \pm 2.80$ \\
\hline 27 & 6-Methyl hept-5-en-2-one & $\mathrm{KE}$ & 11.08 & $0.55 \pm 0.09$ & \\
\hline 28 & Ethyl $(E)$-2-hexenoate & ES & 11.31 & & $1.09 \pm 0.21$ \\
\hline 29 & Hexan-1-ol & $\mathrm{OH}$ & 11.60 & $3.75 \pm 1.71$ & $14.66 \pm 2.22$ \\
\hline 30 & $\begin{array}{l}\text { 3-Methyl-3-butyl } \\
\text { 3-methylbutanoate }\end{array}$ & ES & 11.96 & & $1.36 \pm 0.26$ \\
\hline 31 & (Z)-3-Hexen-1-ol & $\mathrm{OH}$ & 12.33 & $8.94 \pm 1.86$ & $3.74 \pm 0.25$ \\
\hline 32 & Methyl octanoate & ES & 12.46 & & $0.78 \pm 0.02$ \\
\hline 33 & $\begin{array}{l}\text { Ethyl } \\
\text { 3-hydroxy-3-methylbutanoate }\end{array}$ & ES & 12.95 & & $15.28 \pm 2.48$ \\
\hline 34 & $\begin{array}{l}\text { Ethyl } \\
\text { 2-hydroxy-3-methylbutanoate }\end{array}$ & ES & 13.30 & & $1.61 \pm 1.61$ \\
\hline 35 & Ethyl octanoate & ES & 13.60 & $10.72 \pm 0.85$ & $114.54 \pm 10.25$ \\
\hline 36 & 1-Octen-3-ol & $\mathrm{OH}$ & 14.00 & & $1.08 \pm 0.17$ \\
\hline 37 & 3-Methylbutyl hexanoate & ES & 14.20 & $5.63 \pm 0.73$ & $78.48 \pm 9.96$ \\
\hline 38 & Ethyl (Z)-4-octenoate & ES & 14.51 & $0.22 \pm 0.22$ & $13.41 \pm 0.80$ \\
\hline 39 & 2-Ethylhexan-1-ol & $\mathrm{OH}$ & 14.98 & $0.91 \pm 0.91$ & $73.36 \pm 6.36$ \\
\hline 40 & Dodecanal & AL & 15.20 & & $3.05 \pm 1.41$ \\
\hline 41 & Benzaldehyde & AL & 15.48 & & $4.93 \pm 0.43$ \\
\hline 42 & Ethyl 3-hydroxybutanoate & ES & 15.55 & $0.74 \pm 0.74$ & \\
\hline 43 & Propanoic acid & $\mathrm{FA}^{6}$ & 15.96 & $5.64 \pm 1.33^{*}$ & $7.50 \pm 0.90 *$ \\
\hline 44 & Ethyl 2-hydroxypropanoate & ES & 16.19 & & $44.48 \pm 7.50$ \\
\hline 45 & 1-Octanol & $\mathrm{OH}$ & 16.67 & $0.70 \pm 0.70$ & $14.53 \pm 15.01$ \\
\hline 46 & Octyl acetate & ES & 16.68 & & $9.11 \pm 9.11$ \\
\hline 47 & 2-Methylpropanoic acid & FA & 16.70 & $1.62 \pm 1.62$ & \\
\hline 48 & Aristolene & $\mathrm{TE}$ & 16.95 & $3.08 \pm 1.58$ & $40.70 \pm 6.53$ \\
\hline 49 & Methyl benzoate & ES & 17.87 & $8.09 \pm 1.24 *$ & $10.69 \pm 5.95 *$ \\
\hline 50 & 6-Methylheptan-1-ol & $\mathrm{OH}$ & 18.02 & & $22.30 \pm 3.11$ \\
\hline 51 & Butanoic acid & FA & 18.10 & $0.97 \pm 0.97$ & \\
\hline 52 & 6-Methyloctan-1-ol & $\mathrm{OH}$ & 18.32 & & $9.80 \pm 5.01$ \\
\hline
\end{tabular}


Table 1. Cont.

\begin{tabular}{|c|c|c|c|c|c|}
\hline No & Compound & $\begin{array}{l}\text { Compound } \\
\text { Group }\end{array}$ & RT & $\begin{array}{l}\text { Unripe Fruits, } \\
\text { ng/g/h } \pm \mathrm{SE}^{8}\end{array}$ & $\begin{array}{l}\text { Ripe Fruits, } \\
\text { ng/g/h } \pm \mathrm{SE}\end{array}$ \\
\hline 53 & Acetophenone & KE & 18.51 & $1.17 \pm 1.17$ & \\
\hline 54 & Ethyl decanoate & ES & 18.54 & & $16.93 \pm 1.93$ \\
\hline 55 & Ethyl benzoate & ES & 18.94 & $17.39 \pm 3.15$ & $79.04 \pm 9.30$ \\
\hline 56 & Methylbutyl benzoate & ES & 18.97 & $1.02 \pm 1.02$ & \\
\hline 57 & Ethyl (Z)-4-decenoate & ES & 19.03 & $2.44 \pm 2.44$ & $13.58 \pm 2.21$ \\
\hline 58 & 3-Methylbutyl octanoate & $\mathrm{ES}$ & 19.04 & & $28.66 \pm 14.74$ \\
\hline 59 & Unknown & & 19.38 & & $2.36 \pm 1.32$ \\
\hline 60 & Unknown & & 19.78 & & $3.26 \pm 2.21$ \\
\hline 61 & Germacrene D & $\mathrm{TE}$ & 19.98 & & $0.86 \pm 0.51$ \\
\hline 62 & Heptadecane & $\mathrm{AK}^{7}$ & 20.17 & $2.32 \pm 2.32$ & \\
\hline 63 & Unknown & & 20.22 & & $10.61 \pm 5.57$ \\
\hline 64 & $(E, E)$ - $\alpha$-Farnesene & $\mathrm{TE}$ & 20.94 & $4.80 \pm 1.07$ & $10.83 \pm 0.82$ \\
\hline 65 & Methyl salicylate & ES & 21.29 & $8.47 \pm 0.28$ & $25.05 \pm 2.57$ \\
\hline 66 & Ethyl phenylacetate & ES & 21.60 & & $4.26 \pm 0.47$ \\
\hline 67 & 2-Methylpropyl benzoate & ES & 21.71 & & $0.34 \pm 0.34$ \\
\hline 68 & Octadecane & AK & 22.47 & & $2.06 \pm 1.06$ \\
\hline 69 & (E)-Geranyl acetone & $\mathrm{KE}$ & 23.16 & $0.4 \pm 0.03$ & $1.75 \pm 0.96$ \\
\hline 70 & 2-Phenylethanol & $\mathrm{OH}$ & 24.32 & & $23.75 \pm 11.93$ \\
\hline 71 & 3-Methylbutyl benzoate & ES & 24.31 & $48.83 \pm 3.69$ & $142.55 \pm 16.00$ \\
\hline 72 & Heptanoic acid & FA & 25.13 & & $13.13 \pm 4.24$ \\
\hline 73 & 3-Methylbutyl salicylate & ES & 26.93 & & $1.65 \pm 1.12$ \\
\hline 74 & Eugenol & TE & 32.35 & & $0.82 \pm 0.52$ \\
\hline 75 & Hydroxymethylfurfural & AL & 34.90 & & $0.69 \pm 0.36$ \\
\hline \multirow[t]{2}{*}{76} & Benzyl benzoate & ES & 36.94 & & $0.75 \pm 0.38$ \\
\hline & Total & & & $485.1 \pm 93.85$ & $2588.07 \pm 440.78$ \\
\hline
\end{tabular}

${ }^{1} \mathrm{AL}$, aldehyde, ${ }^{2} \mathrm{OH}$, alcohol, ${ }^{3} \mathrm{ES}$, ester, ${ }^{4} \mathrm{KE}$, ketone, ${ }^{5} \mathrm{TE}$, terpenoid, ${ }^{6} \mathrm{FA}$, fatty acid, ${ }^{7} \mathrm{AK}$, alkane; ${ }^{8} \mathrm{SE}-$ standard error of mean; ${ }^{*}$ amounts of volatile organic compound emitted by sea buckthorn fruit at two ripening stages didn't differ significantly (Mann-Whitney U test, $p>0.05$ ).

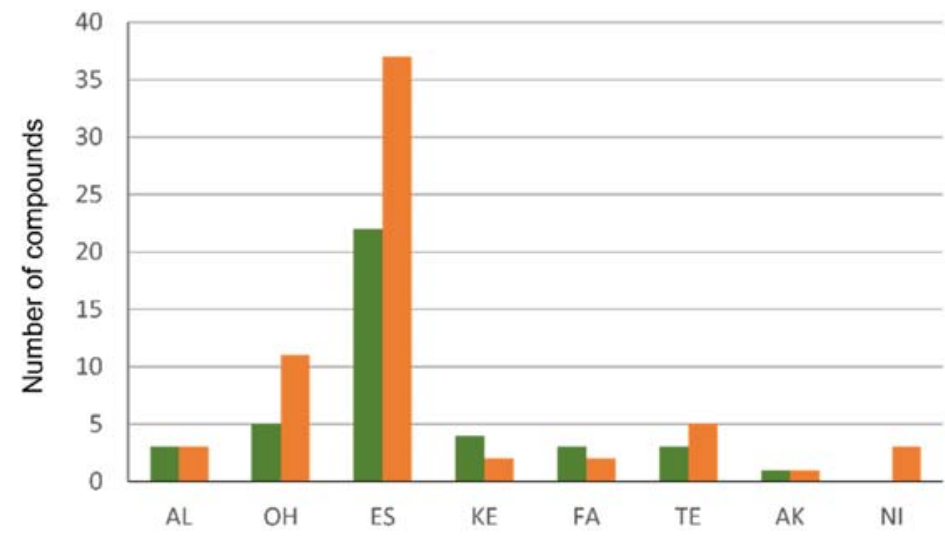

Figure 2. Chemical diversity of the volatile blends produced by sea buckthorn Hippophae rhamnoides berries at two ripening stages: green columns, unripe berries and orange columns, ripe berries. Functional group of volatiles: AL—aldehyde; $\mathrm{OH}$-alcohol; ES—ester; KE-ketone; FA—fatty acid; TE-terpenoid; AK—alkane; NI—not identified.

As many as 35 volatile compounds were found in ripe sea buckthorn fruit emissions only (Table 1). The identified volatiles belonged to five groups of compounds: esters (19 compounds), alcohols (6 compounds), terpenes (4 compounds), aldehydes (3 compounds), and one acid. Among these compounds, esters and alcohols were the most abundant: four esters ethyl 2-methylpropanoate, 3-methylbutyl 2-methylbutanoate, ethyl 2-hydroxypropanoate, and 3-methylbutyl octanoate as well as alcohol 2-methylpropanol were detected in volatile emission at concentrations higher than $25 \mathrm{ng} / \mathrm{g} / \mathrm{h}$. 
The amounts of 2-pentanone, (E)- $\beta$-ocimene, 3-hydroxy-2-butanone, methyl 2-hydroxy2-methylbutanoate, propanoic acid, and methyl benzoate did not differ significantly $(p>0.05)$ in unripe and ripe fruits. (Z)-3-hexenol was detected at significantly higher amounts in unripe compared to ripe fruits $(p<0.05)$, while 21 compounds were emitted at significantly higher amounts $(p<0.05)$ by ripe compared to unripe fruits (Table 1$)$.

\subsection{Olfactory Active Compounds of the Sea Buckthorn Fruits}

GC-EAD analysis of the headspace samples from the sea buckthorn fruits at two ripening stages revealed the presence of at least 26 compounds in unripe soft green fruits and 27 compounds in ripe fruits eliciting the electrophysiological antennal responses both of males and females of $R$. batava (Figure 3). The volatiles of the fruits at two ripening stages differed qualitatively in the single compound only, i.e., 3-methylbutyl 2-methylpropionate, which was found in ripe fruits only (Figure 3).

Table 2. EAD active volatiles identified in headspace of sea buckthorn Hippophae rhamnoides fruits at two ripening stages (unripe fruits and ripe fruits) and their electroantennographic activity to fruit flies Rhagoletis batava.

\begin{tabular}{|c|c|c|c|c|c|c|c|c|c|c|}
\hline \multirow[b]{2}{*}{ No ${ }^{1}$} & \multirow[b]{2}{*}{ Compound } & \multirow[b]{2}{*}{$\mathrm{CG}^{2}$} & \multirow[b]{2}{*}{$\mathrm{RI}^{6}$} & \multirow[b]{2}{*}{$\mathrm{RT}^{7}$} & \multicolumn{2}{|c|}{ Unripe Fruits } & \multicolumn{2}{|c|}{ Ripe Fruits } & \multicolumn{2}{|c|}{ EAG Activity } \\
\hline & & & & & Peak Area 8 & $\begin{array}{c}\text { Peak Area } \\
\%\end{array}$ & Peak Area & $\begin{array}{c}\text { Peak Area } \\
\%\end{array}$ & Females & Males \\
\hline 5 & Ethyl butanoate & $\mathrm{ES}^{3}$ & 1015 & 4.31 & $8.64 \pm 1.26$ & $3.06 \pm 0.14$ & $26.59 \pm 2.61$ & $2.66 \pm 0,04$ & $5^{9}(5) 10$ & $4(4)$ \\
\hline 6 & Ethyl 2-methylbutanoate & ES & 1037 & 4.66 & $28.89 \pm 4.62$ & $10.18 \pm 0.43$ & $\begin{array}{c}129.75 \pm \\
13.20\end{array}$ & $12.98 \pm 0.17$ & $5(5)$ & $4(4)$ \\
\hline 7 & Ethyl 3-methylbutanoate & ES & 1056 & 4.98 & $11.09 \pm 1.33$ & $3.95 \pm 0.09$ & $\begin{array}{l}147.06 \pm \\
14.85\end{array}$ & $14.71 \pm 0.16$ & $5(5)$ & $4(4)$ \\
\hline 10 & Ethyl pentanoate & ES & 1122 & 6.27 & $0.46 \pm 0.09$ & $0.16 \pm 0.01$ & $1.02 \pm 0.15$ & $0.10 \pm 0.01$ & 4(5) & 4(4) \\
\hline 13 & 3-Methylbutyl 2-methylpropionate & ES & 1185 & 7.69 & 0 & 0 & $2.88 \pm 0.27$ & $0.29 \pm 0.001$ & 3(5) & 2(4) \\
\hline 14 & 3-Methylbutan-1-ol & $\mathrm{OH}^{4}$ & 1204 & 8.16 & $8.97 \pm 0.72$ & $3.24 \pm 0.20$ & $26.49 \pm 2.55$ & $2.65 \pm 0.25$ & 5(5) & $4(4)$ \\
\hline 16 & Ethyl 3-methyl-2-butenoate & ES & 1211 & 8.32 & $0.71 \pm 0.09$ & $0.25 \pm 0.01$ & $2.93 \pm 0.37$ & $0.29 \pm 0.03$ & $5(5)$ & $4(4)$ \\
\hline 17 & Ethyl hexanoate & ES & 1232 & 8.80 & $\begin{array}{c}118.03 \pm \\
17.34\end{array}$ & $41.75 \pm 0.52$ & $\begin{array}{c}384.05 \pm \\
38.10\end{array}$ & $38.42 \pm 0.14$ & 5(5) & $4(4)$ \\
\hline 18 & (E)- $\beta$-ocimene & $\mathrm{TE}^{5}$ & 1242 & 9.03 & $28.67 \pm 4.29$ & $10.14 \pm 0.34$ & $35.23 \pm 3.47$ & $3.52 \pm 0.02$ & 5(5) & $4(4)$ \\
\hline 19 & 3-Methylbutyl butanoate & ES & 1258 & 9.43 & $0.23 \pm 0.05$ & $0.08 \pm 0.01$ & $2.20 \pm 0.23$ & $0.22 \pm 0.02$ & $4(5)$ & $4(4)$ \\
\hline 21 & 3-Methylbutyl 2-methylbutanoate & ES & 1271 & 9.75 & $2.17 \pm 0.37$ & $0.76 \pm 0.02$ & $17.76 \pm 1.76$ & $1.78 \pm 0.01$ & 5(5) & $4(4)$ \\
\hline 22 & 3-Methylbutyl 3-methylbutanoate & ES & 1289 & 10.22 & $5.40 \pm 0.65$ & $1.97 \pm 0.33$ & $45.83 \pm 4.54$ & $4.59 \pm 0.05$ & 5(5) & $4(4)$ \\
\hline 23 & Methyl 2-hydroxy-2-methylbutanoate & $\mathrm{ES}$ & 1294 & 10.36 & $0.90 \pm 0.14$ & $0.32 \pm 0.01$ & $1.62 \pm 0.24$ & $0.16 \pm 0.01$ & $5(5)$ & $\begin{array}{l}7(7) \\
4(4)\end{array}$ \\
\hline 25 & Propyl hexanoate & ES & 1309 & 10.74 & $1.77 \pm 0.54$ & $0.60 \pm 0.13$ & $0.97 \pm 0.10$ & $0.10 \pm 0.001$ & $4(5)$ & $4(4)$ \\
\hline 26 & Ethyl heptanoate & ES & 1325 & 11.11 & $3.79 \pm 0.57$ & $1.34 \pm 0.01$ & $4.86 \pm 0.50$ & $0.49 \pm 0.004$ & 5(5) & $4(4)$ \\
\hline 28 & Ethyl $(E)$-2-hexenoate & ES & 1342 & 11.52 & $1.12 \pm 0.21$ & $0.39 \pm 0.02$ & $0.54 \pm 0.06$ & $0.05 \pm 0.001$ & 4(5) & $4(4)$ \\
\hline 30 & 3-Methyl-3-butenyl 3-methylbutanoate & ES & 1368 & 12.16 & $0.02 \pm 0.004$ & $0.01 \pm 0.001$ & $0.05 \pm 0.02$ & $0.01 \pm 0.002$ & 4(5) & 3(4) \\
\hline 32 & Methyl octanoate & ES & 1383 & 12.54 & $3.20 \pm 0.68$ & $1.11 \pm 0.08$ & $1.77 \pm 0.14$ & $0.18 \pm 0.02$ & $5(5)$ & $4(4)$ \\
\hline 35 & Ethyl octanoate & $\mathrm{ES}$ & 1428 & 13.65 & $9.73 \pm 1.57$ & $3.45 \pm 0.25$ & $67.61 \pm 6.79$ & $6.76 \pm 0.11$ & $5(5)$ & $\begin{array}{l}7(7) \\
4(4)\end{array}$ \\
\hline 36 & 1-Octen-3-ol & $\mathrm{OH}$ & 1446 & 14.08 & $0.12 \pm 0.05$ & $0.04 \pm 0.01$ & $0.07 \pm 0.01$ & $0.01 \pm 0.001$ & $5(5)$ & $4(4)$ \\
\hline 38 & Ethyl (Z)-4-octenoate & ES & 1462 & 14.48 & $1.61 \pm 0.24$ & $0.58 \pm 0.05$ & $5.63 \pm 0.33$ & $0.58 \pm 0.08$ & $5(5)$ & $\begin{array}{l}(7) \\
4(4)\end{array}$ \\
\hline 39 & 2-Ethylhexan-1-ol & $\mathrm{OH}$ & 1484 & 15.05 & $2.67 \pm 0.80$ & $0.99 \pm 0.32$ & $2.65 \pm 0.29$ & $0.26 \pm 0.004$ & 5(5) & $3(4)$ \\
\hline 42 & Ethyl 3-hydroxybutanoate & ES & 1512 & 15.72 & $0.39 \pm 0.06$ & $0.14 \pm 0.002$ & $1.24 \pm 0.13$ & $1.24 \pm 0.13$ & $5(5)$ & $4(4)$ \\
\hline 55 & Ethyl benzoate & ES & 1644 & 18.7 & $32.44 \pm 5.50$ & $11.45 \pm 0.57$ & $48.52 \pm 4.74$ & $4.85 \pm 0.03$ & $5(5)$ & $\begin{array}{l}\text { tit) } \\
4(4)\end{array}$ \\
\hline 66 & Ethyl phenylacetate & ES & 1763 & 21.28 & $0.38 \pm 0.07$ & $0.14 \pm 0.03$ & $2.35 \pm 0.27$ & $0.23 \pm 0.01$ & $5(5)$ & 2(4) \\
\hline 69 & (E)-Geranyl acetone & $\mathrm{TE}$ & 1849 & 23.08 & $5.40 \pm 1.00$ & $1.91 \pm 0.20$ & $3.19 \pm 0.47$ & $0.32 \pm 0.03$ & $4(5)$ & $2(4)$ \\
\hline 71 & 3-Methylbutyl benzoate & ES & 1890 & 23.93 & $5.50 \pm 0.88$ & $1.99 \pm 0.33$ & $36.72 \pm 3.60$ & $3.67 \pm 0.05$ & 3(5) & $2(4)$ \\
\hline
\end{tabular}

${ }^{1}$ No is the compound number as indicated in Table 1 and Figure $3 ;{ }^{2} \mathrm{CG}$, compound group; ${ }^{3} \mathrm{ES}$, ester; ${ }^{4} \mathrm{OH}$, alcohol, ${ }^{5} \mathrm{TE}$, terpene; ${ }^{6} \mathrm{RI}$, retention index (polar DB-Wax fused silica capillary column $30 \mathrm{~m} \times 0.25 \mathrm{~mm}$ i.d., $0.25 \mu \mathrm{m}$ film thickness); ${ }^{7}$ Retention time; ${ }^{8}$ Peak area (mean \pm standard error of the mean), the absolute amount expressed as area under the chromatographic peak and have to be read as numbers times 10,$000 ;{ }^{9}$ number of antennae responded, ${ }^{10}$ number of antennae tested.

Ester ethyl hexanoate was the most abundant compound in the headspace of sea buckthorn fruits of both ripening stages; it accounted for about $40 \%$ of the mixture of EADactive compounds (Table 2). Ethyl 2-methylbutanoate, $(E)$ - $\beta$-ocimene, and ethyl benzoate were the compounds each accounting for more than $10 \%$ of the mixture of EAG-active compounds in the unripe fruit emission, while, in the ripe fruits, such compounds were ethyl 2-methylbutanoate and ethyl 3-methylbutanoate. It should also be mentioned that nine compounds in the unripe fruit emission each accounted for $1-5 \%$ of the mixture of EAD active compounds, while, in the ripe fruits, there were nine similar compounds, one of which, i.e., ethyl octanoate, accounted for almost 7\% of the EAG active emission. Each of 13 and 15 volatiles isolated from the emissions of unripe and ripe fruits, respectively, composed less than $1 \%$ of all EAD active compounds (Table 2). 


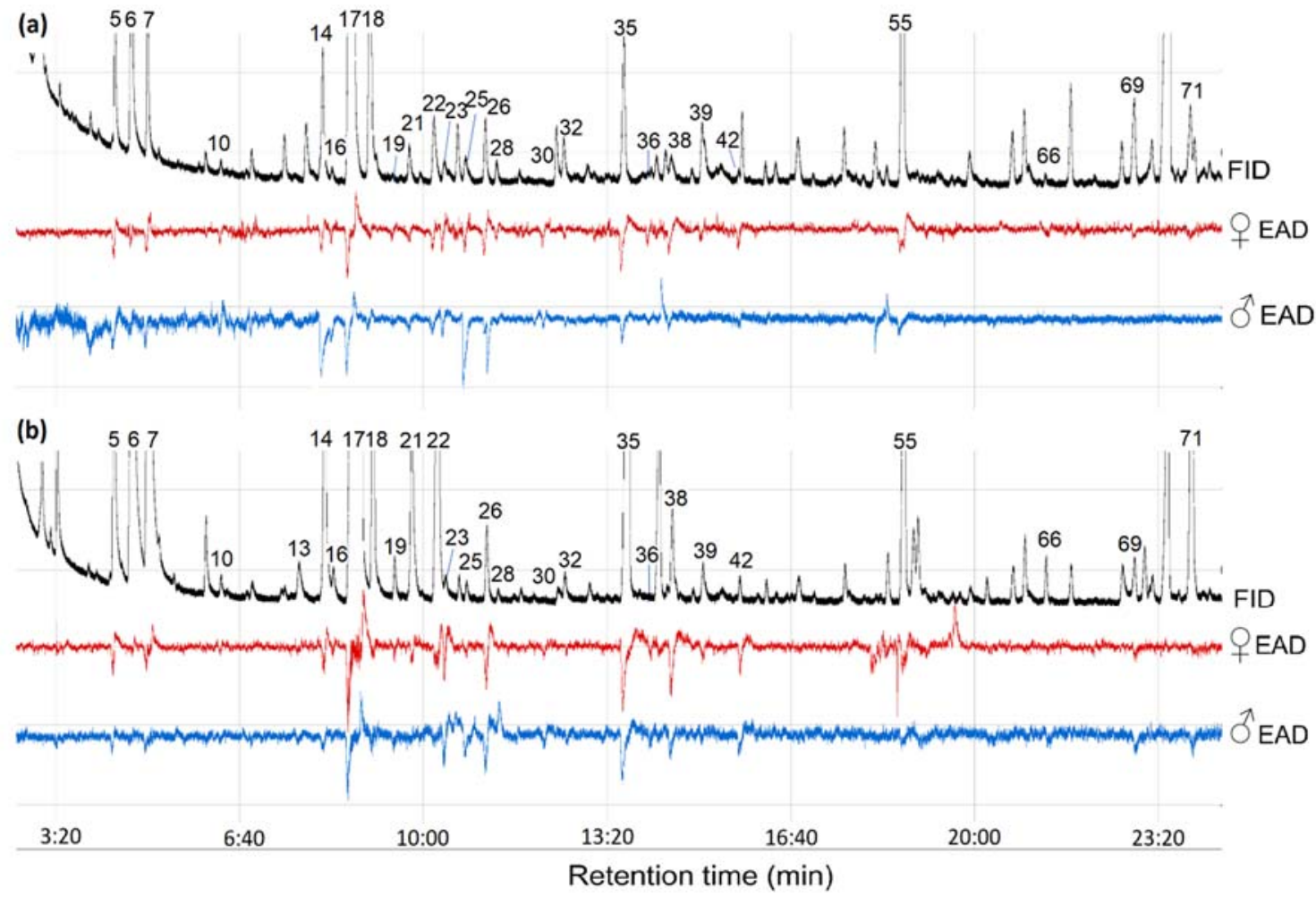

Figure 3. Representative GC-EAD recordings of Rhagoletis batava female and male responses to Hippophae rhamnoides fruits odours at two ripening stages: unripe soft green fruits (a) and ripe orange fruits (b). FID, flame ionization detector; EAD, electroantennographic detector; DB-Wax capillary column (30 m $\times 0.25 \mathrm{~mm} \times 0.25 \mu \mathrm{m}$; Agilent Technologies, Santa Clara, CA, USA). FID peaks are numbered according to Tables 1 and 2.

Esters were the most abundant type of volatiles composing $84 \%$ and $93 \%$ of all EAD active compounds in the emissions from unripe and ripe fruits, respectively. In addition, EAD responses of $R$. batava flies were elicited by three alcohols, which constituted $4.3 \%$ and $2.9 \%$ of the active compounds, as well as by two terpenes that composed $12.1 \%$ and $3.8 \%$ of the EAD active mixture in unripe and ripe fruit emissions, respectively (Table 2).

\section{Discussion}

It is known that plant volatiles act as important chemical signals for a few Rhagoletis species in host-plant selection and could be used as attractants or synergists in integrated pest management [19-24]. However, the compounds released by sea buckthorn H. rhamnoides fruits and perceived by $R$. batava fruit flies, including their changes during the fruit ripening process, were unknown.

In this study, we revealed, for the first time, that the attractiveness of the odour of host-plant fruits to R. batava males and females differs. For males, only ripe fruits are attractive, while females prefer green soft fruits that are not ripe yet. For females, green soft fruits are equally attractive as semi-ripe ones. In contrast to males that were attracted to ripe fruits, females preferred semi-ripe versus ripe fruits. We assume that such a choice is predetermined by the search for the most suitable fruits for egg-laying.

Thermal desorption GC-MS analysis revealed that various esters prevailed in the volatile blends of ripe sea buckthorn fruits. Volatiles of this chemical group composed $57 \%$ of identified compounds and 8 volatiles of 14 compounds trapped at the amounts over $100 \mathrm{ng} / \mathrm{g} / \mathrm{h}$ of fresh fruit weight were esters.

Structurally, the most abundant esters of sea buckthorn fruits are characterised by ethyl moiety and butanoic acid, 2-methylbutanoic acid, 3-methylbutanoic acid, hexanoic acid, octanoic acid, as well as benzoic acid representing the fatty acid part of ester. Our 
data are consistent with the previous reports that volatile profiles of homogenised ripe sea buckthorn fruits were quantitatively and qualitatively dominated by these esters [25-28]. We were able to find only one publication reporting the composition of volatile compounds trapped from intact sea buckthorn fruits. The study by Zapałowska et al. [29] showed that the volatile profiles of ripe fruits were comprised of 8 esters and the same number of volatiles bearing terpene moiety only. A small number of volatiles registered was probably due to a very short period of collection and different chromatographic analysis compared to our study.

A substantial quantitative and qualitative difference of volatile emissions was recorded in unripe and ripe fruits. It is a general trend that fruits release larger amounts of volatiles at the ripe stage compared to the unripe stage [30]. In our study, the total amount of trapped volatiles increased five times compared to emissions from unripe and ripe sea buckthorn fruits. The most pronounced changes in the volatile profiles during ripening of buckthorn fruits have been attributed to the increased number and amount of esters and decline of green leaf volatiles, i.e., (Z)-3-hexanal, (E)-2-hexanal, and (Z)-3-hexenyl acetate. Similar changes have been reported for the number of fruits [31-34].

In order to be able to look for volatile compounds that could lead to the behavioural reactions of the fruit flies, we analysed volatile compounds that they are capable of smelling. GC-EAD analysis of headspace volatiles either of unripe or ripe sea buckthorn fruits revealed 26 compounds in nonripe fruits and 27 compounds in ripe ones. The compounds elicited olfactory responses in antennae, both of males and females.

Among flies from the genus, Rhagoletis persistent EAG responses alongside behavioural activity were recorded in the apple maggot fly, $R$. pomonella. Depending on the host race of the flies and host species, the flies perceived from 5 to 11 volatiles emitted by fruits [35]. Analogous to it, 8 and 9 EAG-active volatiles from snowfruit host fruits were registered in R. mendax [36] and R. zephyria [23], respectively. In $R$. completa, 21 compounds elicited EAG responses [37] and 14 in $R$. cerasi [38]. When comparing this data with the data we recorded for $R$. batava fruit flies, it should be noted that the flies of this species smell the broadest spectrum of the host-plant fruit volatiles. That circumstance makes it more difficult to search for kairomone attractants.

In R. batava, a single EAG active component 3-methylbutyl 2-methylpropionate (peak number 5 in Table 2 and Figure 3) was recorded in ripe fruits only. In unripe fruits, the compound was absent (or its amount was below the detection threshold). EAG responses to the volatile from ripe fruits were very low (Figure 3); thus, we assume the compound should hardly be attributed to important ones, involved in the choice between ripe and unripe fruits.

The sampling of volatiles for the determination of olfactory active compounds was aimed to collect a sufficient amount of volatiles for multiple GC-EAD runs as well as to make stock solutions ensuring as little variation as possible between GC-EAD replicates. Due to the long sampling duration, the use of the solvent to release the volatiles from absorbent followed by concentration of samples affected the ratio between components, which was less accurate compared to the sampling of fruit-released volatiles aiming to quantify the volatiles as accurately as possible. Due to the short collection time and lower release rate from fruits, some volatiles in unripe fruit samples remained below the detection limit in the thermo desorption-GC-MS method compared to the collection of volatiles for GC-EAD experiment.

Difference both in the ratio and in the concentrations of components was obvious when comparing the composition of volatiles emitted by fruits of different ripeness stages. One can assume that these differences could be explored by $R$. batava flies when differentiating fruit attractiveness. There were 17 EAD-active compounds that evoked persistent EAD responses in all tested antennae. Most of these compounds belonged to esters. Butanoic acid moiety found in 8 volatiles was the most abundant fatty acid moiety of these esters, followed by three octanoates, as well as hexanoate, heptanoate, and benzoate, while ethanol 
dominated among alcohol moiety composing the esters. In addition to esters, there were two alcohols 3-methylbutanol and 1-octen-3-ol, and a single terpene (E)- $\beta$-ocimene.

At least 10 EAD-active compounds found in the sea buckthorn fruit emissions, such as ethyl butanoate, ethyl 2-methylbutanoate, ethyl 3-methylbutanoate, 3-methylbutan-1ol, ethyl hexanoate, 3-methylbutyl 2-methylbutanoate, 3-methylbutyl 3-methylbutanoate, and ethyl octanoate, have been reported as compounds released by yeasts related to sea buckthorn fruits $[39,40]$, which, therefore, could be produced by microbiota found on the surface of fruits.

The results we obtained indicated that, among 27 volatile compounds perceived by $R$. batava fruit flies, at least 17 are candidates for kairomone attractants of the pest.

Author Contributions: Conceptualization, V.B. and R.M.; methodology, L.B.-Č., D.A., S.R. and J.B.; software, S.R. and L.B.-Č.; validation, L.B.-Č., R.M., S.R., V.A. and V.B.; formal analysis, L.B.-Č., S.R., V.A. and J.B.; investigation, L.B.-Č., S.R., V.A., D.A. and J.B.; resources, V.B. and R.M.; data curation, L.B.-Č., S.R. and V.A.; writing—original draft preparation, L.B.-Č.; writing—review and editing, L.B.-Č., R.M. and V.B.; visualization, L.B.-Č. and S.R.; supervision, R.M. and V.B.; project administration, L.B.-Č.; funding acquisition, R.M. All authors have read and agreed to the published version of the manuscript.

Funding: This research was funded by European Social Fund, grant number 09.3.3-LMT-K-712-010099 under a grant agreement with the Research Council of Lithuania (LMTLT).

Institutional Review Board Statement: Not applicable.

Informed Consent Statement: Not applicable.

Data Availability Statement: Not applicable.

Acknowledgments: We thank G. Bumbulytè and R. Čepulyte for helping to collect the fruit fly puparia and to carry out behavioural test.

Conflicts of Interest: The authors declare no conflict of interest. The funders had no role in the design of the study; in the collection, analyses, or interpretation of data; in the writing of the manuscript, or in the decision to publish the results.

\section{References}

1. Li, T.S.C. Taxonomy, natural distribution and botany. In Sea Buckthorn (Hippophae rhamnoides L.): Production and Utilization; Li, T.S.C., Beveridge, T., Eds.; NRC Research Press: Ottawa, ON, USA, 2003; pp. 7-11.

2. Li, T.S.C.; Beveridge, T. Sea Buckthorn (Hippophaè rhamnoides L.): Production and Utilization; NRC Research Press: Ottawa, ON, USA, 2003.

3. Kalia, R.K.; Singh, R.; Rai, M.K.; Mishra, G.P.; Singh, S.R.; Dhawan, A.K. Biotechnological interventions in sea buckthorn (Hippophae L.): Current status and future prospects. Trees 2011, 25, 559-575. [CrossRef]

4. Li, T.S.C.; Schroeder, W.R. Sea buckthorn (Hippophae rhamnoides L.): A multipurpose plant. Horttechnology 1996, 6, 370-380. [CrossRef]

5. Gâtlan, A.M.; Gutt, G. Sea buckthorn in plant based diets. An analytical approach of sea buckthorn fruits composition: Nutritional value, applications, and health benefits. Int. J. Environ. Res. Public Health 2021, 18, 8986. [CrossRef] [PubMed]

6. Brūvelis, A. Feasibility Study for Trapping of the Sea Buckthorn Flies and Biological Control of Wilt. In Proceedings of the EuroWorkS On Air 3 "Seabuckthorn Field Technologies, Including Pests and Diseases Control", Eurasia, Online, 23 November 2021; Available online: https:/ / euroworks.online/wp/projects / (accessed on 29 December 2021).

7. Höhne, F.; Gießmann, H.J. Ein neuer Schädling bedroht den Sanddornanbau massives Auftreten in Versuchen der Landesforschungsanstalt 2013! Info-Blatt 2013, 22, 280-285.

8. Stalažs, A. New records of some dipterans (Diptera: Cecidomyidae, Tephritidae) in North-Eastern Lithuania. Zool. Ecol. 2014, 24, 55-57. [CrossRef]

9. Stalažs, A.; Balalaikins, M. Country checklist of Rhagoletis Loew (Diptera: Tephritidae) for Europe, with focus on R. batava and its recent range expansion. Proc. Latvian Acad. Sci. Section B 2017, 71, 103-110. [CrossRef]

10. Shamanskaya, L.D. Bioecology of the sea-buckthorn fly (Rhagoletis batava obscuriosa Kol.) and pest control treatment in Altai. In Proceedings of the 3rd European Workshop on Sea Buckthorn EuroWorkS2014, Natural Resources Institute Finland, Naantali, Finland, 14-16 October 2014; pp. 7-20.

11. Toth, M.; Lerche, S.; Holz, U.; Kerber, A.; Henning, R.; Voigt, E.; Kelemen, D. Addition of synthetic feeding attractant increases catches of Rhagoletis batava Hering and Carpomyia schineri Loew. in fluorescent yellow sticky traps. Acta Phytopathol. Entomol. Hung. 2016, 51, 69-76. [CrossRef] 
12. Leblanc, L.; Vargas, R.I.; Rubinoff, D. A comparison of nontarget captures in BioLure and liquid protein food lures in Hawaii. Proc. Hawaii. Entomol. Soc. 2010, 42, 15-22.

13. Yee, W.L. Ammonium carbonate loss rates from lures differentially affect trap captures of Rhagoletis indifferens (Diptera: Tephritidae) and non-target flies. Can. Entomol. 2017, 149, 241-250. [CrossRef]

14. Būda, V.; Blažytè-Čereškienè, L.; Radžiutè, S.; Apšegaitè, V.; Schultz, S.; Stamm, P.; Aleknavičius, D.; Mozūraitis, R. Male-produced (-)- $\delta$-heptalactone as pheromone of the fruit fly Rhagoletis batava (Diptera: Tephritidae), a pest of sea buckthorn fruits. Insects $\mathbf{2 0 2 0}$ 11, 138. [CrossRef]

15. Aldrich, J.R.; Bartelt, R.J.; Dickens, J.C.; Knight, A.L.; Light, D.M.; Tumlinson, J.H. Insect chemical ecology research in the United States Department of Agriculture-Agricultural Research Service. Pest Manag. Sci. 2003, 59, 777-787. [CrossRef] [PubMed]

16. Reddy, G.V.P.; Guerrero, A. Interactions of insect pheromones and plant semiochemicals. Trends Plant Sci. $2004,9,253-261$. [CrossRef] [PubMed]

17. Sablon, L.; Dickens, J.C.; Haubruge, É.; Verheggen, F.J. Chemical ecology of the colorado potato beetle, Leptinotarsa decemlineata (Say) (Coleoptera: Chrysomelidae), and potential for alternative control methods. Insects 2013, 4, 31-54. [CrossRef] [PubMed]

18. Scolari, F.; Valerio, F.; Benelli, G.; Papadopoulos, N.T.; Vaníčková, L. Tephritid fruit fly semiochemicals: Current knowledge and future perspectives. Insects 2021, 12, 408. [CrossRef] [PubMed]

19. Fein, B.L.; Reissig, W.H.; Roelofs, W.L. Identification of apple volatiles attractive to the apple maggot, Rhagoletis pomonella. J. Chem. Ecol. 1982, 8, 1473-1487. [CrossRef]

20. Zhang, A.J.; Linn, C.; Wright, S.; Prokopy, R.; Reissig, W.; Roelofs, W. Identification of a new blend of apple volatiles attractive to the apple maggot, Rhagoletis pomonella. J. Chem. Ecol. 1999, 25, 1221-1232. [CrossRef]

21. Prokopy, R.J.; Papaj, D.R. Behavior of flies of the genera Rhagoletis, Zonosemata, and Carpomya (Trypetinae: Carpomyina). In Fruit Flies (Tephritidae): Phylogeny and Evolution of Behavior; Aluja, M., Norrbom, A.L., Eds.; CRC Press: Boca Raton, FL, USA, 2001; pp. 219-252.

22. Cha, D.H.; Yee, W.L.; Goughnour, R.B.; Sim, S.B.; Powell, T.H.Q.; Feder, J.L.; Linn, C.E., Jr. Identification of host fruit volatiles from domestic apple (Malus domestica), native black hawthorn (Crataegus douglasii) and introduced ornamental hawthorn (C. monogyna) attractive to Rhagoletis pomonella flies from the western United States. J. Chem. Ecol. 2012, 38, 319-329. [CrossRef]

23. Cha, D.H.; Olsson, S.B.; Yee, W.L.; Goughnour, R.B.; Hood, G.R.; Mattsson, M.; Schwarz, D.; Feder, J.L.; Linn, C.E., Jr. Identification of host fruit volatiles from snowfruit (Symphoricarpos albus), attractive to Rhagoletis zephyria flies from the western United States. J. Chem. Ecol. 2017, 43, 188-197. [CrossRef]

24. Quilici, S.; Atiama-Nurbel, T.; Brévault, T. Plant odors as fruit fly attractants. In Trapping and the Detection, Control, and Regulation of Tephritid Fruit Flies; Shelly, T., Epsky, N., Jang, E., Reyes-Flores, J., Vargas, R., Eds.; Springer: Dordrecht, The Netherlands, 2014; pp. 119-144. [CrossRef]

25. Tiitinen, K.; Hakala, M.; Kallio, H. Headspace volatiles from frozen fruits of sea buckthorn (Hippophae rhamnoides L.) varieties. Eur. Food Res. Technol. 2006, 223, 455-460. [CrossRef]

26. Socaci, S.A.; Socaciu, C.; Tofana, M.; Rati, I.V.; Pintea, A. In-tube extraction and GC-MS analysis of volatile components from wild and cultivated sea buckthorn (Hippophae rhamnoides L. ssp Carpatica) fruit varieties and juice. Phytochem. Anal. 2013, 24, 319-328. [CrossRef]

27. Leung, G.S.; Marriott, R. Year to year variation in sea buckthorn juice volatiles using headspace solid phase microextraction. Flavour Fragr. J. 2016, 31, 124-136. [CrossRef]

28. Ma, X.Y.; Yang, W.; Marsol-Vall, A.; Laaksonen, O.; Yang, B.R. Analysis of flavour compounds and prediction of sensory properties in sea buckthorn (Hippophae rhamnoides L.) fruits. Int. J. Food Sci. Technol. 2020, 55, 1705-1715. [CrossRef]

29. Zapalowska, A.; Matlok, N.; Zardzewialy, M.; Piechowiak, T.; Balawejder, M. Effect of ozone treatment on the quality of sea buckthorn (Hippophae rhamnoides L.). Plants 2021, 10, 847. [CrossRef] [PubMed]

30. Baietto, M.; Wilson, A.D. Electronic-nose applications for fruit identification, ripeness and quality grading. Sensors 2015, 15, 899-931. [CrossRef]

31. Jayanty, S.; Song, J.; Rubinstein, N.M.; Chong, A.; Beaudry, R.M. Temporal relationship between ester biosynthesis and ripening events in bananas. J. Am. Soc. Hortic. Sci. 2002, 127, 998-1005. [CrossRef]

32. Jetti, R.R.; Yang, E.; Kurnianta, A.; Finn, C.; Qian, M.C. Quantification of selected aroma-active compounds in strawfruits by headspace solid-phase microextraction gas chromatography and correlation with sensory descriptive analysis. J. Food Sci. 2007, 72, S487-S496. [CrossRef]

33. Li, G.; Jia, H.; Li, J.; Wang, Q.; Zhang, M.; Teng, Y. Emission of volatile esters and transcription of ethylene- and aroma-related genes during ripening of 'Pingxiangli' pear fruit (Pyrus ussuriensis Maxim). Sci. Hortic. 2014, 170, 17-23. [CrossRef]

34. Wang, M.Y.; MacRae, E.; Wohlers, M.; Marsh, K. Changes in volatile production and sensory quality of kiwifruit during fruit maturation in Actinidia deliciosa Hayward' and A. chinensis Hort16A'. Postharvest Biol. Technol. 2011, 59, 16-24. [CrossRef]

35. Tait, C.; Batra, S.; Ramaswamy, S.S.; Feder, J.L.; Olsson, S.B. Sensory specificity and speciation: A potential neuronal pathway for host fruit odour discrimination in Rhagoletis pomonella. Proc. R. Soc. B 2016, 283, 20162101. [CrossRef]

36. Lugemwa, F.N.; Lwande, W.; Bentley, M.D.; Mendel, M.J.; Alford, A.R. Volatiles of wild bluefruit, Vaccinium angustifolium: Possible attractants for the bluefruit maggot fruit fly, Rhagoletis mendax. J. Agric. Food Chem. 1989, 37, 232-233. [CrossRef]

37. Hamersky, W. Electrophysiological and Behavioral Responses of Walnut Husk flies to Walnut Volatiles. Master's Thesis, The California State University, Long Beach, CA, USA, August 1996. 
38. Būda, V.; Radžiutè, S.; Apšegaitè, V.; Blažytè-Čereškienè, L.; Čepulytė, R.; Bumbulytè, G.; Mozūraitis, R. Electroantennographic and behavioural responses of European cherry fruit fly, Rhagoletis cerasi, to the volatile organic compounds from sour cherry, Prunus cerasus, fruit. Insects 2022, 13, 114. [CrossRef]

39. Mozūraitis, R.; Aleknavičius, D.; Vepštaitè-Monstavičè, I.; Stanevičienè, R.; Emami, S.N.; Apšegaitè, V.; Radžiutė, S.; BlažytèČereškienè, L.; Servienè, E.; Būda, V. Hippophae rhamnoides fruit related Pichia kudriavzevii yeast volatiles modify behaviour of Rhagoletis batava flies. J. Adv. Res. 2020, 21, 71-77. [CrossRef] [PubMed]

40. Lukša, J.; Vepštaitè-Monstavičè, I.; Apšegaitè, V.; Blažytè-Čereškienė, L.; Stanevičienè, R.; Strazdaitè-Žielienè, Ž.; Ravoitytè, B.; Aleknavičius, D.; Būda, V.; Mozūraitis, R.; et al. Fungal microbiota of sea buckthorn fruits at two ripening stages and volatile profiling of potential biocontrol yeasts. Microorganisms 2020, 8, 456. [CrossRef] [PubMed] 